\title{
Endoleak Detection after Endovascular Aortic Aneurysm Repair Using Ultrasound Based on Nanoscale Bubble Contrast Agents and Their Effects on Vascular Smooth Muscle Cell Proliferation and Migration
}

\author{
Siying Pei, ${ }^{1}$ Yao Feng $\mathbb{D}^{2},{ }^{2}$ Shuqing Fang, ${ }^{3}$ Song Jin, ${ }^{4}$ Dongxu Fan $\mathbb{D}^{4},{ }^{4}$ Fanxu Song ${ }^{\mathbb{D}},{ }^{5}$ \\ and Hanrui Wang $\mathbb{B}^{4}$ \\ ${ }^{1}$ College of Basic Medicine, Jiamusi University, China \\ ${ }^{2}$ The First Affiliated Hospital of Jiamusi University, China \\ ${ }^{3}$ Department of Nephrology, Jiamusi Central Hospital, China \\ ${ }^{4}$ Department of Vascular Surgery, The First Affiliated Hospital of Jiamusi University, China \\ ${ }^{5}$ The Third Affiliated Hospital of Jiamusi University, China
}

Correspondence should be addressed to Hanrui Wang; whr496196111@163.com

Received 8 April 2021; Accepted 25 April 2021; Published 11 May 2021

Academic Editor: Songwen Tan

Copyright (c) 2021 Siying Pei et al. This is an open access article distributed under the Creative Commons Attribution License, which permits unrestricted use, distribution, and reproduction in any medium, provided the original work is properly cited.

\begin{abstract}
Abdominal aortic aneurysm (AAA) is the most common vascular disease that causes disability and death. Its morbidity is relatively subtle, and the mortality rate is high. Clinically, endovascular aortic aneurysm repair (EVAR) has gradually become the primary treatment of AAA due to its unique advantages such as low trauma and low incidence of short-term complications. However, the outcome of EVAR is greatly compromised by the possible occurrence of endoleaks. Contrast-enhanced ultrasound (CEUS) is a promising alternative technique to detect endoleaks following EVAR due to lack of exposure to ionizing radiation. Traditional ultrasound contrast agents with an overlarge size (microscale) leading to reluctant accumulation in target organs and instability trigger the requirement of nanoscale contrast agents that enter tumor tissues through the enhanced permeability and retention effect. In this study, we used ultrasound based on nanoscale bubble contrast agents to evaluate endoleak detection after endovascular aortic aneurysm repair and analyzed the effects of nanoscale bubble contrast agents on vascular smooth muscle cell (VSMC) proliferation and migration. Among 52 AAA patients a month following EVAR, there were 16 cases of endoleaks after EVAR detected by nanobubble contrast-enhanced ultrasound, including 6 cases of type I endoleak (1 case of type Ia endoleak and 5 cases of type Ib endoleak), 7 cases of type II endoleak, and 3 cases of type III endoleak; there were 12 cases of endoleak after EVAR detected by computed tomography angiography (CTA), including 6 cases of type I endoleak (1 case of type Ia and 5 cases of type Ib), 5 cases of type II endoleak, and 1 case of type III endoleak. Six months after EVAR, 3 cases of type III endoleak were detected by both nanobubble contrast-enhanced ultrasound and CTA. Vascular smooth muscle cells (VSMCs) used for in vitro experiments were subjected to ultrasound irradiation and platelet-derived growth factor (PDGF) treatment with or without the addition of nanobubble contrast agents. After high-intensity and long-term irradiation $\left(0.75 \mathrm{~W} / \mathrm{cm}^{2}\right.$ and $1 \mathrm{~W} / \mathrm{cm}^{2}$ irradiation for $120 \mathrm{~s}$ and $150 \mathrm{~s}$, respectively) by ultrasound with or without the addition of nanobubble contrast agents, PDGF-induced VSMC migration was inhibited $(P<0.01)$. Low-intensity and short-term ultrasound irradiation did not differ PDGF-induced VSMC migration $(P>0.05)$, but $0.5 \mathrm{~W} / \mathrm{cm}^{2}$ and $90 \mathrm{~s}$ ultrasound irradiation could significantly inhibit PDGF-induced VSMC migration without the addition of nanobubble contrast agents $(P<0.05)$. When VSMCs were irradiated at $1,0.75,0.5$, and $0.35 \mathrm{~W} / \mathrm{cm}^{2}$ for $30 \mathrm{~s}$ to $150 \mathrm{~s}$, ultrasound irradiation with or without the addition of nanobubble contrast agents remarkably reduced PDGF-induced VSMC proliferation, as evidenced by reduced OD values $(P<0.05)$. In conclusion, ultrasound based on nanoscale bubble contrast agents is an effective alternative detection method for the occurrence of AAA patients who are not suitable for CTA.
\end{abstract}




\section{Introduction}

Abdominal aortic aneurysm (AAA) is a disease with a serious increase in morbidity year by year. The main risk is rupture, and the total mortality of ruptured tumor is $80 \%$ to $90 \%$ [1]. Endovascular aortic aneurysm repair (EVAR) has become the main method for clinical treatment of diseases such as abdominal aortic aneurysm and abdominal aortic dissection [2]. Compared with open surgery, perioperative morbidity, mortality, and average length of stay of EVAR patients have obvious advantages [3]. However, with the passage of time, due to factors such as the reintervention rate of EVAR and the need for lifelong monitoring, the advantages of EVAR are becoming increasingly unclear. One of the factors that seriously affect the reintervention rate is the occurrence of endoleaks. At the same time, the presence of an endoleak increases the risk of tumor growth and rupture after EVAR [4]. Therefore, in the intracavitary treatment of EVAR, it is very important for the prognosis of patients to find the endoleak and solve the endoleak accordingly [5].

Contrast-enhanced ultrasound (CEUS) has been used more and more in the follow-up of EVAR in recent years. It is a new type of noninvasive imaging technology. It is based on conventional ultrasound examination and is injected by intravenous ultrasound [6]. Agents are used to enhance the human body's blood flow scattering signal, thereby merging the resolution, sensitivity, and specificity of a high ultrasound diagnosis. The ultrasound contrast agent can be kept in the blood pool of the human body for a certain period, thereby enhancing the visualization effect of blood vessels and realtime observation of blood flow perfusion in the lesion area. With the development of nanotechnology and molecular biology, nanotype contrast agents, due to their outstanding characteristics of small molecules and strong penetrating power, will strongly promote the expansion of ultrasound molecular imaging to the extravascular field.

This study is based on the advantages of noninvasive, nonradiative, easy to operate, and good repeatability of nanobubble contrast agents, which can actively gather in the tumor tissue, enhance tumor tissue visualization, improve the sensitivity and accuracy of tumor detection, and achieve extravascular detection of lesions and ultrasound molecular imaging, compared with the examination results of ultrasound nanobubbles in endoleaks after intraluminal repair of patients with AAAs, showing that the ultrasonic imaging of nanobubbles that detected complications after intraluminal repair of AAAs has a good positive rate, and further analysis of the effects of nanobubble ultrasound contrast agents in the proliferation and migration of vascular smooth muscle cells can provide a reliable diagnosis basis for the clinic and has extremely high clinical application value.

\section{Materials and Methods}

2.1. Nanobubble Contrast Agents. The preparation of nanobubbles is mainly to prepare the mixed contrast agent of microbubbles and nanobubbles by the film hydration method and then separate the pure nanobubbles by centrifugation, static, or filter methods or to add surfactants in the production process; in addition to increasing the operation process, it will also increase the potential possibility of sample pollution and reduce the production of nanobubbles, and by adjusting the dosage and preparation conditions of the liposome, we can make a suitable liposome film, which can directly prepare pure nanobubbles, and test the indicators of nanobubbles [7]. Figure 1 shows the preparation of nanovesicles and their state in blood vessels.

AAA is divided into a true one and a pseudo one according to the composition structure of the aneurysm [8]. True AAA is the permanent limited expansion of the abdominal aorta, the blood vessel wall is intact, the blood is still in the vascular lumen, and the wall of the true AAA has a threelayer structure of arterial walls. Pseudo-AAA can be regarded as an injury to the wall of the arterial tube, causing blood to flow from the lumen of the artery to the outside, and a tumor-like structure is formed by wrapping the external tissue [9]. The wall of the pseudo-AAA is compressed by the surrounding organization structure. In clinical work, the most common causes are atherosclerosis and degenerative changes. These two causes may exist at the same time, or they may exist separately $[10,11]$.

As shown in Figure 2, when the diameter of a solid tumor grows to about $2 \mathrm{~mm}$, angiogenic factors are released to induce angiogenesis, the micro vessels formed by the tumor tissue are irregularly shaped and swollen, the endothelial cells are loosely arranged, and a gap of 380-780 $\mathrm{nm}$ can be formed between the endothelial cells [12]. The return of the lymphatic system is missing [13]. These vascular characteristics promote the high permeability and retention effect of lipid particles in the tumor tissue, which is what we call the EPR effect $[14,15]$. It is generally believed that ultrasound contrast agents with a diameter of less than $7 \mathrm{~nm}$ can pass through the gap of the inner wall of the tumor blood vessel and enter the surrounding tumor tissue.

2.2. Contrast Process of Nanobubble Agent. The process of imaging tumor tissue with a nanobubble ultrasound contrast agent is shown in Figure 3. Compared with other types of nanoscale ultrasound contrast agents, the core of the nanobubble ultrasound contrast agent is a gas, which has a stronger backscattering ability in the ultrasonic sound field, and due to the compressibility of the gas, the nanobubbles will have a diameter. It has a nonlinear acoustic characteristic, so that it can better develop the target tissue, and it can use the nanobubble-sensitive ultrasound contrast imaging technology with its nonlinear acoustic characteristic [16, 17]. In addition, the nanobubble ultrasound contrast agent has the unique characteristics of many surface-active centers, high surface reactivity, long half-life in vivo, strong adsorption capacity, and not being degraded by various enzymes in the body and cells. Research applications in imaging and therapeutics have broad prospects [18].

Studies have shown that when different examination methods are used for patients with AAAs, different degrees of atherosclerosis and calcification can be seen on the walls of AAAs, indicating that there is also atherosclerosis in patients with AAAs and degenerative changes [19, 20]. Animal experiments have confirmed that long-term feeding of 


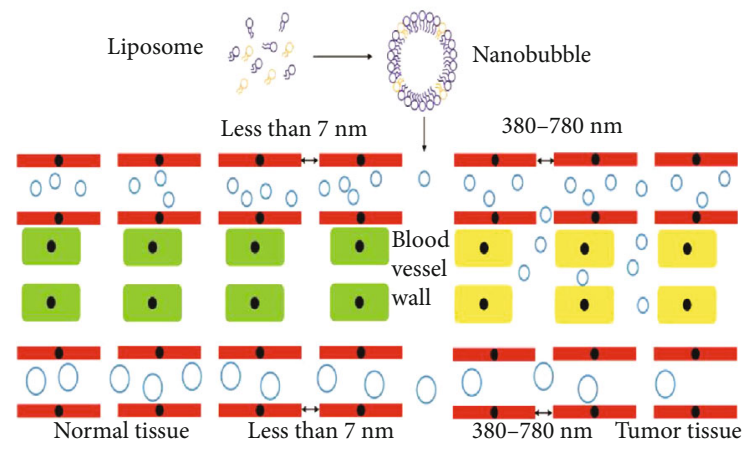

Figure 1: Preparation of nanobubbles and their states in blood vessels.

an arteriosclerosis diet to primates can easily form aneurysms, proving that atherosclerosis plays an important role in the formation of AAAs [21]. Some scholars believe that degenerative changes have a greater effect, because in AAA specimens, metalloproteinases are overexpressed, which is an elastase, which can degrade elastic fibers in the arterial wall, resulting in compliance of the arterial wall and the decrease in the formation of aneurysms; in addition, increased metalloproteinase inhibitors and low expression of antiproteolytic enzymes also indirectly confirm the role of degenerative degeneration in the formation of AAAs $[19,22]$.

2.3. Patients. From June 2018 to June 2019, a total of 52 patients, including 39 males and 13 females, aged 56-85 years, and with an average age of $68.5 \pm 6.72$ years, were diagnosed with AAA and underwent EVAR in our hospital. The abdominal aorta had various degrees of atherosclerosis and multiple spot block formations. All patients underwent computed tomography angiography (CTA) and ultrasound examination at the first month and the sixth month after surgery to determine whether there was an endoleak. If there was an endoleak, the location and the type of the endoleak were determined by CTA and nanobubble ultrasound contrast examination.

2.4. Inclusion Criteria. Using the Chinese vascular surgery guidelines, the local expansion of the artery is more than $50 \%$ of the outer diameter of the normal artery. AAA is generally defined as the outer diameter of the abdominal aorta that exceeds $3 \mathrm{~cm}$. This numerical standard is calculated based on the average value of the normal population, but there are also studies that show that the outer diameter of the abdominal aorta is positively correlated with age, sex, body surface area, etc.; the normal outer diameter of the abdominal aorta will vary greatly according to gender, age, and body surface area, so the outer diameter of the abdominal aorta that exceeds $3 \mathrm{~cm}$ which is defined as an active abdominal aneurysm is not very rigorous; so in clinical work, AAA usually refers to the outer diameter of the artery that exceeds $50 \%$ of the outer diameter of the adjacent normal artery. In this study, the outer diameter of the AAA was measured and compared with the outer diameter of the neighboring normal artery, when more than 50\% were included in the study.
2.5. Ultrasound Contrast Examination. The patient was fasted and placed in a supine position. Firstly, the position, size, range, and thrombus of the AAA were observed by two-dimensional grayscale ultrasound and whether there was any change compared with that before surgery. Secondly, the position and presence of the stent graft were observed, the blood flow in the stent graft was observed by color Doppler technology, the abnormal blood flow in and around the stent graft was monitored in real time, and finally, the spectrum Doppler technology was used to analyze the hemodynamic of the abnormal site to determine whether there is an endoleak; if an abnormal blood flow signal appears in the external tumor cavity of the stent graft, it is judged as positive for an endoleak $[23,24]$. For patients with endoleaks, rough classification is based on the location of the endoleak and related blood flow. The classification of an endoleak was defined as previously reported by White et al. [25]. The surgical society agrees that when the data is collated, it will be classified according to the more stringent and standardized ultrasound-typing guidelines. Each patient is examined by two senior physicians or deputy chief physicians, and the analysis results are summarized and analyzed. For the two doctors who have different examination results, the chief physician conducts a third examination combined with the first two physicians checking the results and gives the final diagnosis.

2.6. Cell Treatment. Human aortic VSMCs (Shanghai Cell Bank, Chinese Academy of Sciences, Shanghai, China) was maintained in DMEM with $10 \%$ fetal bovine serum (FBS, Gibco, USA), $100 \mu \mathrm{g} / \mathrm{ml}$ streptomycin, and $100 \mathrm{IU} / \mathrm{ml}$ penicillin in a humidified environment in the presence of $5 \%$ $\mathrm{CO}_{2}$. VSMCs used for in vitro experiments were subjected to ultrasound irradiation and platelet-derived growth factor (PDGF) treatment with or without addition of nanobubble contrast agents. In brief, VSMCs were placed onto a 6-well culture plate and serum-starved for $48 \mathrm{~h}$. The culture plate was placed in a $37^{\circ} \mathrm{C}$ water bath, and $10 \mathrm{ng} / \mathrm{ml}$ PDGF (Gibco, USA) was added to the culture solution [26]. Following $24 \mathrm{~h}$ of culture, ultrasound irradiation was performed. The ultrasound probe was fixed at a distance of $3 \mathrm{~mm}$ from the bottom of the culture plate, with a frequency of $1 \mathrm{MHz}$ and indicated irradiation times $(0 \mathrm{~s}, 30 \mathrm{~s}, 60 \mathrm{~s}, 90 \mathrm{~s}, 120 \mathrm{~s}$, and $150 \mathrm{~s})$ under the intensity of $0.35 \mathrm{~W} / \mathrm{cm}^{2}, 0.5 \mathrm{~W} / \mathrm{cm}^{2}, 0.75 \mathrm{~W} / \mathrm{cm}^{2}$, and $1.0 \mathrm{~W} / \mathrm{cm}^{2}$. As for addition of nanobubble contrast agents, the culture medium with the addition of PDGF addition was added again with $1 \mu \mathrm{l} / \mathrm{m}$ nanobubble contrast agents and incubated for $24 \mathrm{~h}$, followed by parallel ultrasonic irradiation.

2.7. MTT Assay. VSMC proliferation was determined using an MTT assay. In brief, $24 \mathrm{~h}$ after ultrasonic irradiation, VSMCs $\left(2 \times 10^{3}\right.$ cells/well $)$ were plated into 96 -well plates. The optical density (OD) of each well was measured at $450 \mathrm{~nm}$ using a plate reader (Thermo Scientific, Watertown, USA).

2.8. Transwell Migration Assay. VSMC migration was assessed using a transwell $(8 \mu \mathrm{m}$ pore size), and $24 \mathrm{~h}$ after ultrasonic irradiation, $5 \times 10^{4}$ cells were plated into each well of the upper chamber uncoated with the membrane. The lower chamber was added with the medium containing $10 \%$ 


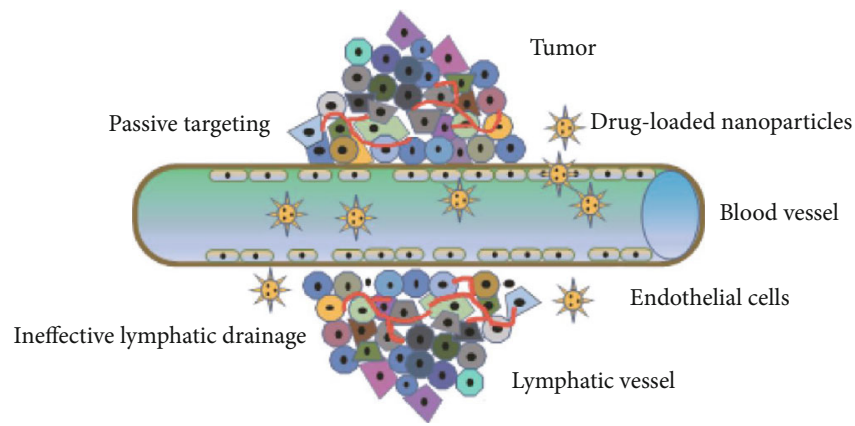

FIGURE 2: Drug-loaded nanoparticles diffuse to the tumor site through the enhanced permeability and retention effect.

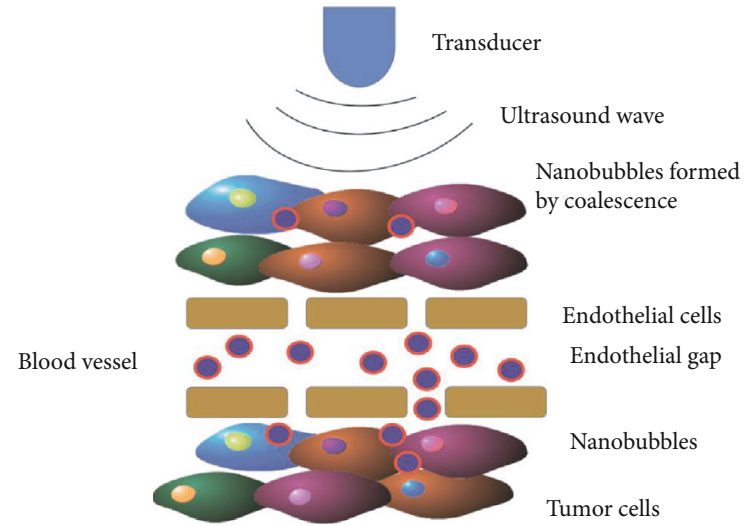

Figure 3: The contrast process of tumor tissues by ultrasound using nanobubble contrast agents.

FBS. After cells were incubated for 24 hours, those that migrated from the upper chamber into the lower chamber were fixed with methanol and stained with $0.1 \%$ crystal violet dye. A microscopic view was captured under an IX71 inverted microscope (OLYMPUS, Olympus Corporation, Tokyo, Japan).

2.9. Statistical Methods. SPSS 26.0 international standard statistical software was used for statistical data analysis, the variable was evaluated as positive for endoleak, and the positive rate of endoleak detected by the two inspection methods was calculated. The McNemar test was used to compare the detection frequency of the two inspection parties. When $P<0.05$, the difference was statistically significant. By calculating the $k$ value and comparing the consistency of ultrasound, according to the Landis and Koch guidelines, the $k$ value represents different consistency in different intervals. When the $k$ value is $0.21-0.40$, it means that the consistency is poor; when the $k$ value is $0.41-0.60$, it means moderate consistency; when the $k$ value is $0.61-0.80$, it means better consistency; and when the $k$ value is $0.81-1.0$, it means excellent consistency.

\section{Results}

3.1. Endoleak Detection a Month and Six Months after EVAR Using Nanobubble Contrast-Enhanced Ultrasound. Among 52 AAA patients, there were 16 cases of endoleak after EVAR detected by nanobubble contrast-enhanced ultrasound,
TABLE 1: Endoleak detection a month after EVAR using nanobubble contrast-enhanced ultrasound.

\begin{tabular}{lccc}
\hline Nanobubble contrast-enhanced & \multicolumn{2}{c}{ CTA } & \multirow{2}{*}{ Total } \\
ultrasound & Positive & Negative & \\
\hline Positive & 11 & 5 & 16 \\
Negative & 3 & 33 & 36 \\
Total & 14 & 38 & 52 \\
\hline
\end{tabular}

including 6 cases of type I endoleak ( 1 case of type Ia endoleak and 5 cases of type Ib endoleak), 7 cases of type II endoleak, and 3 cases of type III endoleak. The detection rate was $30.76 \%$. There were 12 cases of endoleak after EVAR was detected by CTA, including 6 cases of type I endoleak (1 case of type Ia and 5 cases of type Ib), 5 cases of type II endoleak, and 1 case of type III endoleak. The detection rate was $23.08 \%$. A total of 11 cases of endoleak were detected by both nanobubble contrast-enhanced ultrasound and CTA, and the classification of the endoleak was the same. However, 1 case of type Ib endoleak, 3 cases of type II endoleak, and 1 case of type III endoleak were detected by nanobubble contrastenhanced ultrasound, which were not evident by CTA (Table 1). As for the classification of the endoleak, $11.5 \%$ were type I endoleak (1.9\% of type Ia endoleak and $9.6 \%$ of type Ib endoleak), $13.46 \%$ of type II endoleak, and $5.77 \%$ of type III endoleak were detected by nanobubble contrastenhanced ultrasound. The CTA detected $26.92 \%$ of the patients with endoleak of which type I endoleak accounted for $13.46 \%$ including $3.84 \%$ type Ia and $9.6 \%$ type Ib endoleak, type II endoleak accounted for 9.6\%, and type III endoleak accounted for $3.84 \%$ (Table 2). Six months after EVAR, there were 3 cases of type III endoleak that were detected by both nanobubble contrast-enhanced ultrasound and CTA (Table 3). The CTA examination results are the same as the ultrasound examination results.

3.2. Effect of Nanobubble Contrast Agents on PDGF-Induced VSMC Migration. After high-intensity and long-term irradiation $\left(0.75 \mathrm{~W} / \mathrm{cm}^{2}\right.$ and $1 \mathrm{~W} / \mathrm{cm}^{2}$ irradiation for $120 \mathrm{~s}$ and $150 \mathrm{~s}$, respectively) by ultrasound with or without the addition of nanobubble contrast agents, PDGF-induced VSMC migration was inhibited $(P<0.01)$. Low-intensity and short-term ultrasound irradiation did not differ PDGFinduced VSMC migration $(P>0.05)$, but $0.5 \mathrm{~W} / \mathrm{cm}^{2}$ and $90 \mathrm{~s}$ ultrasound irradiation could significantly inhibit 
TABLE 2: Endoleak detection according to classifications a month after EVAR using nanobubble contrast-enhanced ultrasound.

\begin{tabular}{lcccrr}
\hline \multirow{2}{*}{ Result } & \multicolumn{2}{c}{ Type I } & \multirow{2}{*}{ Type II } & \multirow{2}{*}{ Type III } & \multirow{2}{*}{ Type IV } \\
& Ia & Ib & & & \\
\hline Nanobubble contrast-enhanced ultrasound & $1.9 \%$ & $9.6 \%$ & $13.46 \%$ & $5.77 \%$ & 0 \\
CTA & $3.84 \%$ & $9.6 \%$ & $9.6 \%$ & $3.84 \%$ & $0.76 \%$ \\
\hline
\end{tabular}

TABLE 3: Endoleak detection according to classifications six months after EVAR using nanobubble contrast-enhanced ultrasound.

\begin{tabular}{lcccccc}
\hline Time & \multicolumn{2}{c}{ Type I } & \multicolumn{2}{c}{ Type II } & \multicolumn{2}{c}{ Type III } \\
& Ultrasound & CTA & Ultrasound & CTA & Ultrasound & 3 \\
\hline One month & 6 & 6 & 7 & 3 & 3 & 0 \\
Six months & 0 & 0 & 3 & 3 & 0 \\
\hline
\end{tabular}

PDGF-induced VSMC migration without the addition of nanobubble contrast agents $(P<0.05)$. All in all, the inhibition of PDGF-induced VSMC migration was the greatest for $1 \mathrm{~W} / \mathrm{cm}^{2}$ ultrasound irradiation for $150 \mathrm{~s}$ in the presence of nanobubble contrast agents $(P<0.01$, Figures 4 and 5$)$. Figure 6 presents the numbers of migrating VSMC by ultrasound irradiation at $1,0.75,0.5$, and $0.35 \mathrm{~W} / \mathrm{cm}^{2}$ for $30 \mathrm{~s}$ to $150 \mathrm{~s}$ with or without the addition of nanobubble contrast agents.

\subsection{Effect of Nanobubble Contrast Agents on PDGF-Induced} VSMC Proliferation. When VSMCs were irradiated at 1 , $0.75,0.5$, and $0.35 \mathrm{~W} / \mathrm{cm}^{2}$ for $30 \mathrm{~s}$ to $150 \mathrm{~s}$, ultrasound irradiation with or without the addition of nanobubble contrast agents remarkably reduced PDGF-induced VSMC proliferation, as evidenced by reduced OD values $(P<0.05$, Tables $4-7)$. When VSMCs were irradiated at $1 \mathrm{~W} / \mathrm{cm}^{2}$ for $90 \mathrm{~s}$ and $150 \mathrm{~s}$, nanobubble contrast agents remarkably enhanced PDGFinduced VSMC proliferation $(P<0.05)$. When VSMCs were irradiated at $1 \mathrm{~W} / \mathrm{cm}^{2}$ for $30 \mathrm{~s}, 60 \mathrm{~s}$, and $120 \mathrm{~s}$, nanobubble contrast agents remarkably reduced PDGF-induced VSMC proliferation $(P<0.01)$. When VSMCs were irradiated at $0.75 \mathrm{~W} / \mathrm{cm}^{2}, 60 \mathrm{~s}$ of ultrasound irradiation led to the smallest inhibitory effect on PDGF-induced VSMC proliferation $(P<0.05)$, and $90 \mathrm{~s}$ of ultrasound irradiation with the addition of nanobubble contrast agents led to the smallest inhibitory effect on PDGF-induced VSMC proliferation $(P<0.05)$.

\section{Discussion}

In this study, among 52 cases of AAA, 39 were male and 13 were female and the average age was $68.1 \pm 6.68$ years. In the examination of whether there is an endoleak after endovascular repair of AAA, ultrasound also found that the 52 patients had different degrees of atherosclerosis and multiple plaque formations; most of the plaques were mixed echo and strong echo plaques; strong echo plaques and mixed echo plaques showed that the abdominal aorta had different degrees of degeneration, which was the same as the previous research results. In this study, 42 patients with AAA underwent intracavitary repair. There was no significant difference in the detection rate of the endoleak after intracavitary repair of AAA between the two methods. The $k$ value of the two

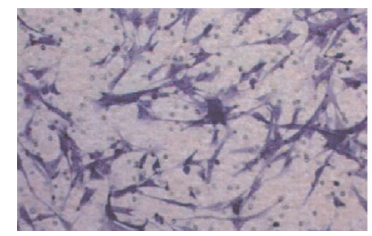

Before ultrasound irradiation

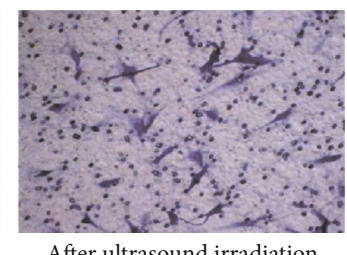

After ultrasound irradiation
FIgURE 4: Representative views of VSMCs migrating from the uncoated upper chamber into the lower one before and after ultrasound irradiation.

inspection methods $(k=0.703)$ is calculated, and it is found that the two inspection methods are highly consistent, the classification of the endoleak is generally based on the location of the extravasation of the contrast agent, but there is lack of information such as the direction of internal blood flow [27, 28]. Different doctors may have different classifications of endoleaks. However, with the help of color Doppler technology, ultrasound can provide more blood flow information, and the classification of endoleaks is based on the relationship between blood flow signal, stent graft, and peripheral blood vessels, and especially for the type II endoleak, it can be traced back according to the branch pipe of the backflow. Although most type II endoleaks do not need to be treated, they still need to be treated when the blood perfusion volume is large and the AAA gradually becomes large. At this time, the artery traced by ultrasound will provide reliable reference for the treatment of coil embolism in the clinical bed. But ultrasound is easy to be interfered by tissue movement to produce false images. Currently, it is necessary for patients to hold their breath. In addition, ultrasound is not satisfied with deep and low velocity blood flow and depends on the technology and experience of the examiner. Although ultrasound has the above limitations, they do not affect that it becomes a reliable method for the follow-up of endoleaks after endovascular repair of AAA.

In the study, we found that the frequency of VSMCs was $1 \mathrm{MHz}$; the sound intensity was $0.35 \mathrm{~W} / \mathrm{cm}^{2}, 0.50 \mathrm{~W} / \mathrm{cm}^{2}$, $0.75 \mathrm{~W} / \mathrm{cm}^{2}$, and $1 \mathrm{~W} / \mathrm{cm}^{2}$; and the time was $20 \mathrm{~s}, 30 \mathrm{~s}, 60 \mathrm{~s}$, $90 \mathrm{~s}, 120 \mathrm{~s}$, and $150 \mathrm{~s}$, after continuous ultrasound irradiation, whether or not micro irradiation was combined. In this study, the number of vascular smooth muscle cell migration increased significantly. It can cause significant migration of 

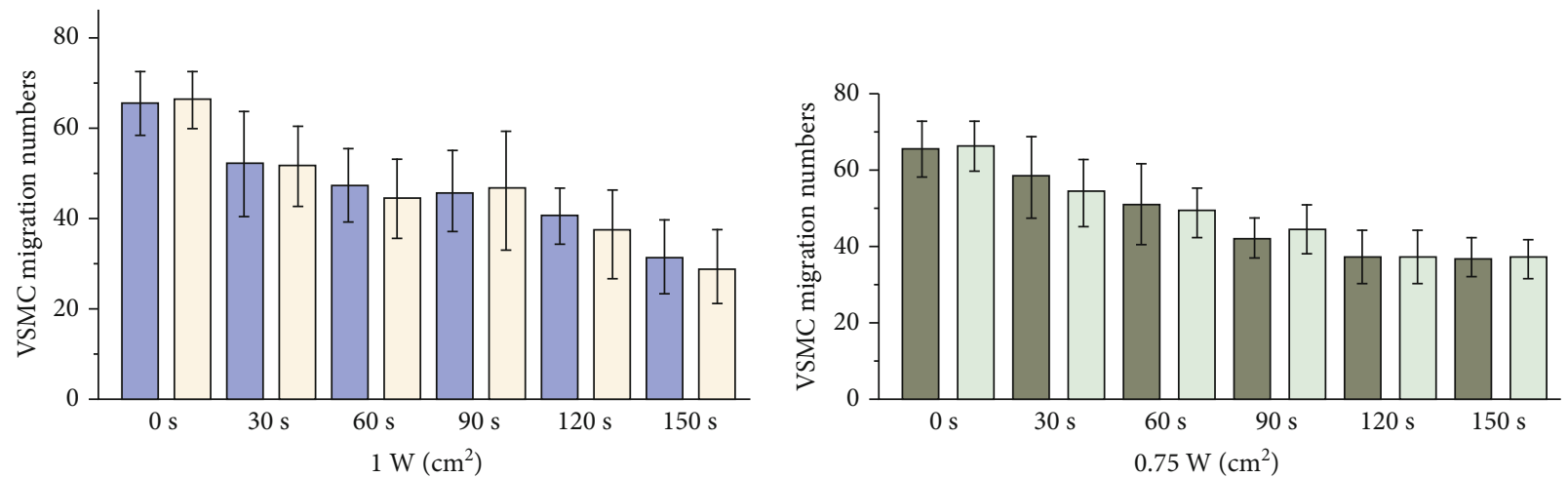

$\square$ Ultrasound irradiation

$\square$ Nanobubble ultrasound irradiation

Ultrasound irradiation

Nanobubble ultrasound irradiation
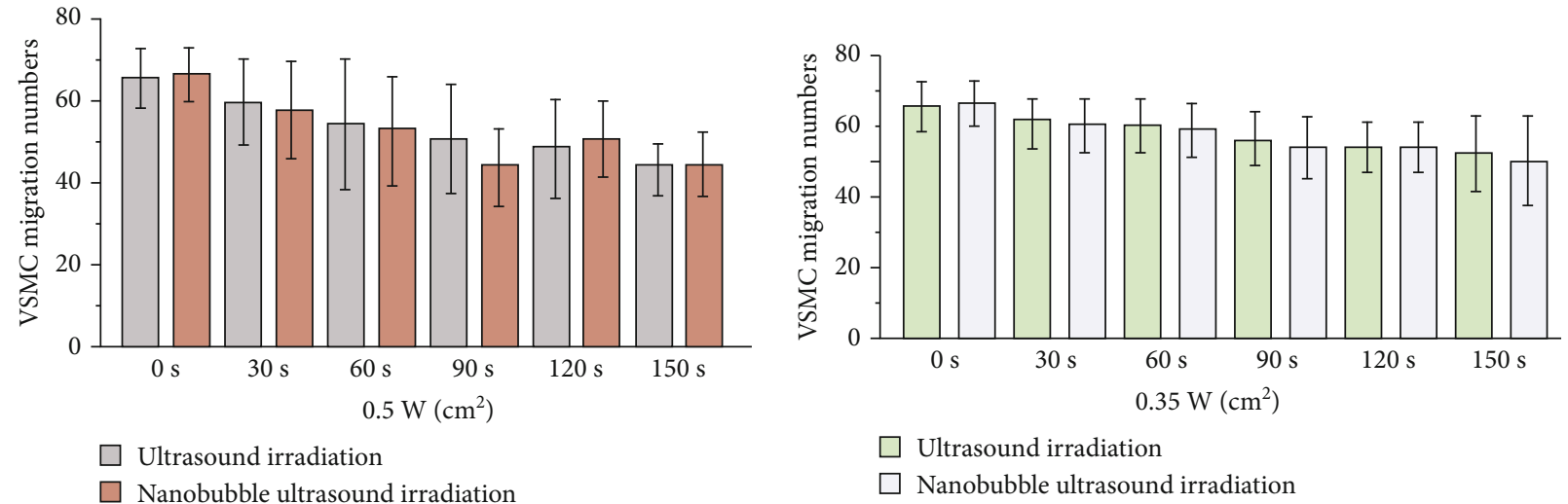

Figure 5: The numbers of PDGF-induced VSMCs under the ultrasound irradiation of $1,0.75,0.5$, and $0.35 \mathrm{~W} / \mathrm{cm}^{2}$ for $30 \mathrm{~s}$ to $150 \mathrm{~s}$ with or without the addition of nanobubble contrast agents.
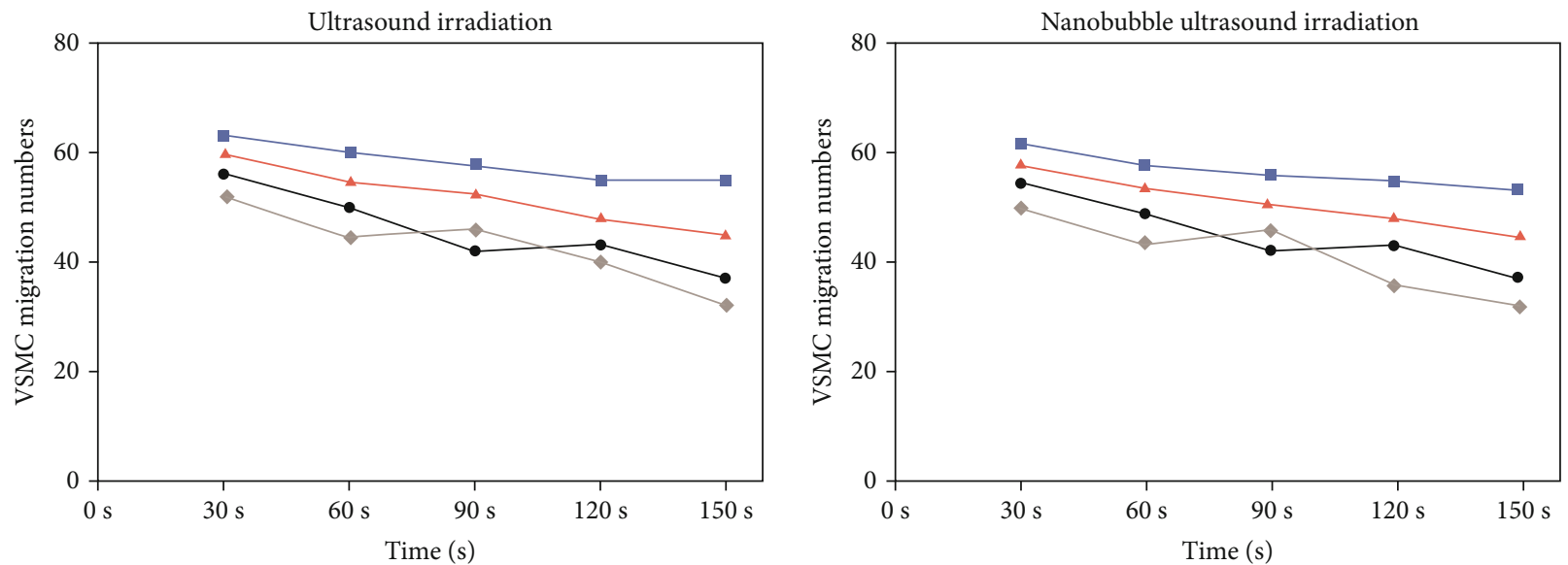

$$
\begin{aligned}
& \rightarrow 0.35 \mathrm{~W} / \mathrm{cm}^{2} \\
& \leftarrow 0.5 \mathrm{~W} / \mathrm{cm}^{2}
\end{aligned}
$$

$\rightarrow 0.75 \mathrm{~W} / \mathrm{cm}^{2}$

$1 \mathrm{~W} / \mathrm{cm}^{2}$

FIGURE 6: The numbers of migrating VSMC by ultrasound irradiation at $1,0.75,0.5$, and $0.35 \mathrm{~W} / \mathrm{cm}^{2}$ for $30 \mathrm{~s}$ to $150 \mathrm{~s}$ with or without addition of nanobubble contrast agents.

VSMCs. Whether or not nanobubble contrast agents were added, PDGF-induced VSMC migration was significantly reduced after high-intensity and long-term ultrasound irradiation, which proves that ultrasound irradiation with a certain intensity and irradiation time or ultrasound irradiation based on nanobubble contrast agents can inhibit the migration of VSMCs in a certain dose.

The biological behavior of VSMCs, such as proliferation and migration, is a dynamic process, which is regulated by many factors including the signal transduction pathway, 
TABLE 4: The OD value of VSMCs under the ultrasound irradiation of $1 \mathrm{~W} / \mathrm{cm}^{2}$ with or without the addition of nanobubble contrast agents.

\begin{tabular}{lcccccc}
\hline Time $(\mathrm{s})$ & 0 & 30 & 60 & 90 & 120 & 150 \\
\hline Ultrasound irradiation group & $0.886 \pm 0.004$ & $0.697 \pm 0.005$ & $0.686 \pm 0.006$ & $0.662 \pm 0.005$ & $0.687 \pm 0.015$ & $0.645 \pm 0.006$ \\
Nanobubble ultrasound irradiation group & $0.886 \pm 0.004$ & $0.685 \pm 0.010$ & $0.663 \pm 0.003$ & $0.697 \pm 0.008$ & $0.665 \pm 0.005$ & $0.673 \pm 0.010$ \\
\hline
\end{tabular}

TABLE 5: The OD value of VSMCs under the ultrasound irradiation of $0.75 \mathrm{~W} / \mathrm{cm}^{2}$ with or without the addition of nanobubble contrast agents.

\begin{tabular}{lcccccc}
\hline Time $(\mathrm{s})$ & 0 & 30 & 60 & 90 & 120 & 150 \\
\hline Ultrasound irradiation group & $0.776 \pm 0.004$ & $0.674 \pm 0.007$ & $0.692 \pm 0.008$ & $0.670 \pm 0.006$ & $0.660 \pm 0.007$ & $0.663 \pm 0.007$ \\
Nanobubble ultrasound irradiation group & $0.776 \pm 0.004$ & $0.678 \pm 0.006$ & $0.674 \pm 0.003$ & $0.682 \pm 0.004$ & $0.662 \pm 0.009$ & $0.667 \pm 0.005$ \\
\hline
\end{tabular}

TABLE 6: The OD value of VSMCs under the ultrasound irradiation of $0.5 \mathrm{~W} / \mathrm{cm}^{2}$ with or without the addition of nanobubble contrast agents.

\begin{tabular}{lcccccc}
\hline Time $(\mathrm{s})$ & 0 & 30 & 60 & 90 & 120 & 150 \\
\hline Ultrasound irradiation group & $0.775 \pm 0.006$ & $0.692 \pm 0.003$ & $0.684 \pm 0.006$ & $0.678 \pm 0.006$ & $0.686 \pm 0.008$ & $0.678 \pm 0.006$ \\
Nanobubble ultrasound irradiation group & $0.775 \pm 0.006$ & $0.686 \pm 0.002$ & $0.682 \pm 0.005$ & $0.683 \pm 0.007$ & $0.682 \pm 0.005$ & $0.675 \pm 0.004$ \\
\hline
\end{tabular}

TABLE 7: The OD value of VSMCs under the ultrasound irradiation of $0.35 \mathrm{~W} / \mathrm{cm}^{2}$ with or without the addition of nanobubble contrast agents.

\begin{tabular}{lcccccc}
\hline Time (s) & 0 & 30 & 60 & 90 & 120 & 150 \\
\hline Ultrasound irradiation group & $0.769 \pm 0.006$ & $0.682 \pm 0.004$ & $0.688 \pm 0.007$ & $0.681 \pm 0.007$ & $0.678 \pm 0.007$ & $0.683 \pm 0.005$ \\
Nanobubble ultrasound irradiation group & $0.769 \pm 0.006$ & $0.695 \pm 0.003$ & $0.686 \pm 0.008$ & $0.692 \pm 0.005$ & $0.685 \pm 0.006$ & $0.692 \pm 0.007$ \\
\hline
\end{tabular}

cytokines, growth factors, vasoactive substances, and extracellular matrix. In this study, the number of VSMC migration increased significantly after the stimulation of PDGF, which indicated that the dose of PDGF in this study could cause VSMC proliferation and migration significantly [29]. Whether an ultrasound contrast agent is added or not, the proliferation and migration of VSMCs are significantly reduced, which prove that ultrasound with a certain intensity and irradiation time can inhibit the proliferation and migration of VSMCs at a certain dose. More importantly, we found that the number of VSMCs in the process of proliferation and migration was gradually reduced with the extension of irradiation time, reaching the lowest point when the sound intensity reached $1 \mathrm{~W} / \mathrm{cm}^{2}$ and the time reached $150 \mathrm{~s}$. First, under the premise of not causing irreversible damage to cells, $1 \mathrm{~W} / \mathrm{cm}^{2}$ and $150 \mathrm{~s}$ are the most obvious conditions for the inhibition of vascular smooth muscle cell migration. Second, such results are caused by the limited sound intensity and time point we selected [30]. If we choose a higher or lower sound intensity and a longer or shorter time, we may find more obvious inhibition, and this is also the limitation of our research. Further research can be carried out through experiments in the future. Similarly, there is a similar situation about the value of VSMCs after ultrasonic treatment with certain intensity and irradiation time. At the same time, in the comparison between the irradiation group and the combination group, we found that the migration number of VSMCs in the irradiation group was lower than that in the ultrasound irradiation group at the same time and sound intensity. We think that this is because the ultrasound contrast agent, as a kind of artificial cavitation nucleus, increased the concentration of the cavitation nucleus in the body and reduced the ultrasound cavitation; the threshold value enhances the cavitation effect and reduces the threshold value of ultrasonic energy needed to produce the cavitation effect $[31,32]$. For the proliferation of VSMCs, in the comparison between the irradiation group and the irradiation group, under the same time and sound intensity, the value of VSMCs in the irradiation group was higher than that in the ultrasound irradiation group, which may be due to the fact that the density of ultrasound nanovesicles as an artificial cavitation nucleus was not enough to reduce the ultrasound cavitation threshold, which can enhance the cavitation effect but cannot reduce the threshold value of the ultrasound energy needed to produce cavitation effect, so the effect of migration inhibition is not as good as that of the microbubble contrast agent.

In this paper, we studied the enhancement of the acoustic intensity and the sensitivity of the detection of the endoleak caused by the nanobubble ultrasound contrast agent. The nanobubble ultrasound contrast agent can scan the blood flow inside the stent graft and the aneurysm cavity from multiple angles and in real time. It is also more sensitive to the capture of some low-speed blood flow signals. The detection effect of the nanobubble ultrasound contrast agent on the endoleak is higher than that of the conventional ultrasound 
effect of CTA and is the same. In the occult endoleak, the detection effect of the endoleak is even higher than that of $\mathrm{CTA}$, and the cost of contrast-enhanced ultrasound is lower, there is no radiation, and the contrast-enhanced ultrasound is metabolized by the lung. Therefore, a more effective examination method is provided for the patients who are not suitable for CTA visit. Due to the lack of large sample data accumulation and analysis, it is believed that through the continuous accumulation and research, nanobubble ultrasound has a broader application prospect in the follow-up of AAA after intracavitary repair.

\section{Data Availability}

The data used to support the findings of this study are included within the article.

\section{Ethical Approval}

All data, models, and code generated or used during the study appear in the submittal complying with the Ethics of Experimentation Statement. The study follows the principles of the Declaration of Helsinki.

\section{Disclosure}

Siying Pei and Yao Feng should be considered as co-first authors.

\section{Conflicts of Interest}

No potential conflict of interest was reported by the authors.

\section{Authors' Contributions}

Siying Pei and Yao Feng contributed equally to this work.

\section{Acknowledgments}

Scientific research business fee project of Heilongjiang Provincial Education Department (Grant: 2016-KYYWF-0586, 2018-KYYWF-0967).

\section{References}

[1] N. Sakalihasan, R. Limet, and O. D. Defawe, "Abdominal aortic aneurysm," Lancet, vol. 365, no. 9470, pp. 1577-1589, 2005.

[2] A. Calero and K. A. Illig, "Overview of aortic aneurysm management in the endovascular era," Seminars in Vascular Surgery, vol. 29, no. 1-2, pp. 3-17, 2016.

[3] A. Patel, R. Edwards, and S. Chandramana, "Surveillance of patient's post-endovascular abdominal aortic aneurysm repair (EVAR), a web-based survey of practice in the UK," Clinical Radiology, vol. 68, no. 6, pp. 580-587, 2016.

[4] A. A. Contador, D. E. D. Siqueira, O. B. Jacobsen et al., "Duplex ultrasound, and computed tomography angiography in the follow-up of endovascular abdominal aortic aneurysm repair: a comparative study," Radiologic Brasilia, vol. 49, no. 4, pp. 229-233, 2016.

[5] M. Serac, I. Marfanoid, A. Tombic, S. Sarac, and M. Bezmarević, "Endovascular repair of ruptured abdominal aortic aneurysm," Vojnosanitetski Pregel, vol. 71, no. 1, pp. 78-82, 2016.

[6] D. A. Vessel, I. S. Shacking, K. V. Bacalao et al., "On the problem of internal optical loss and current leakage in laser heterostructures based on AlGaInAs/InP solid solutions," Semiconductors, vol. 50, no. 9, pp. 1225-1230, 2016.

[7] J. DuPont, A. McCurley, and S. Bende, "Mechanism of aging-associated hypertension: role of smooth muscle cell mineralocorticoid receptor regulation of vascular L-type calcium channels," Journal of Beijing Polytechnic University, vol. 31, no. 31, pp. 399-404, 2016.

[8] C. E. Wells, N. D. Pugh, and J. P. Woodcock, “Abdominal aortic aneurysm detection by common femoral artery Doppler ultrasound waveform analysis," Journal of Medical Engineering \& Technology, vol. 35, no. 1, pp. 34-39, 2016.

[9] M. Serac, I. Marfanoid, A. Tombic, S. Sarac, and M. Bezmarević, "Endovascular repair of ruptured abdominal aortic aneurysm," Vojnosanitetski Pregled, vol. 71, no. 1, pp. 78-82, 2014.

[10] X. L. Li, Q. F. Deng, X. Zhang et al., "The design and implementation of decompression sickness bubble detection system based on dynamic ultrasound images," Journal of Medical Imaging and Health Informatics, vol. 8, no. 5, pp. 900-906, 2016.

[11] S. Fukuda, H. Watanabe, K. Isaura et al., "Multicentre investigations of the prevalence of abdominal aortic aneurysm in elderly Japanese patients with hypertension," Circulation Journal, vol. 79, no. 3, pp. 524-529, 2016.

[12] M. Mell, T. Garg, and L. C. Baker, "Under-utilization of routine ultrasound surveillance after endovascular aortic aneurysm repair," Annals of Vascular Surgery, vol. 34, no. 6, pp. 24-25, 2017.

[13] M. Kuribayashi, "Vascular calcification pathological mechanism and clinical application: role of vascular smooth muscle cells in vascular calcification," Clinical Calcium, vol. 25, no. 5, pp. 661-669, 2016.

[14] T. L. Chen, H. J. Mao, and C. Chen, "The role and mechanism of alpha-klotho in the calcification of rat aortic vascular smooth muscle cells," BioMed Research International, vol. 16, no. 7, 197 pages, 2016.

[15] X. D. Chang, B. H. Zhang, L. H. Li, and Z. C. Feng, "T3 inhibits the calcification of vascular smooth muscle cells and the potential mechanism," American Journal of Translational Research, vol. 8, no. 11, pp. 4694-4704, 2016.

[16] Y. R. Jiang, J. Yang, X. W. Liu, Y. Li, and Q. T. Zeng, "Effects and related mechanism of quercetin on thrombin-induced proliferation and migration of rat vascular smooth muscle cells," Chinese Journal of Cardiovascular Diseases, vol. 44, no. 8, pp. 696-699, 2016.

[17] Y. Wang, H. X. Zhou, and L. Wang, "Changes of calcium mobilization and calcium-sensitization mechanism in vascular smooth muscle cells in hypertension," Chinese Pharmacological Bulletin, vol. 30, no. 2, pp. 287-291, 2016.

[18] J. A. Leopold, "Vascular calcification: mechanisms of vascular smooth muscle cell calcification," Trends in Cardiovascular Medicine, vol. 25, no. 4, pp. 267-274, 2016.

[19] Y. H. Kim, J. H. Han, E. J. Yun et al., "Inhibitory effect of a novel naphthoquinone derivative on proliferation of vascular smooth muscle cells through suppression of platelet-derived growth factor receptor $\beta$ tyrosine kinase," European Journal of Pharmacology, vol. 733, pp. 81-89, 2014. 
[20] B. Tang, X. S. Dong, Z. Wei, H. Q. Qiao, H. C. J. B. Liu, and X. Y. Sun, "Enhanced autophagy by enviroximes contributes to the antiaesthetic mechanisms in vascular smooth muscle cells," Journal of Vascular Research, vol. 51, no. 4, pp. 259268, 2016.

[21] F. Malgorzata and C. M. Shanahan, "ER stress regulates alkaline phosphatase gene expression in vascular smooth muscle cells via an ATF4-dependent mechanism," Bic Research Notes, vol. 11, no. 1, pp. 483-485, 2016.

[22] H. D. Liu, W. Xiong, Q. Y. Liu et al., "Role of CMKLR1 on mouse vascular smooth muscle cells proliferation and related mechanism," Chinese Journal of Cardiovascular Diseases, vol. 44, no. 7, pp. 605-609, 2016.

[23] M. F. Shih, K. H. Pan, and J. Y. Cherng, "Possible mechanisms of di(2-ethylhexyl) phthalate-induced MMP-2 and MMP-9 expression in A7r5 rat vascular smooth muscle cells," International Journal of Molecular Sciences, vol. 16, no. 12, pp. 2880028811, 2016.

[24] R. Sudor, F. Sato, T. Akech, and H. Wachi, "7-Ketocholesterolinduced lysosomal dysfunction exacerbates vascular smooth muscle cell calcification via oxidative stress," Genes to Cells, vol. 20, no. 12, pp. 982-991, 2016.

[25] G. H. White, J. May, R. C. Waugh, X. Chaufour, and W. Yu, "Type III and type IV endoleak: toward a complete definition of blood flow in the sac after endoluminal AAA repair," The European Journal of Vascular and Endovascular Surgery, vol. 5, no. 4, pp. 305-309, 1998.

[26] I. C. Lo, T. M. Lin, L. H. Chou et al., "Ets-1 mediates plateletderived growth factor-BB-induced thrombomodulin expression in human vascular smooth muscle cells," Cardiovascular Research, vol. 81, no. 4, pp. 771-779, 2009.

[27] U. A. Kaysi, M. Basra, G. K. Ozlem et al., "Long-acting progestin-only contraceptives impair endometrial vasculature by inhibiting uterine vascular smooth muscle cell survival," Proceedings of the National Academy of Sciences of the United States of America, vol. 112, no. 16, pp. 5153-5158, 2016.

[28] A. Frismantiene, M. Philippova, P. Erne, and T. J. Resink, "Smooth muscle cell-driven vascular diseases and molecular mechanisms of VSMC plasticity," Cellular Signalling, vol. 52, pp. 48-64, 2016.

[29] D. M. Liu, W. Cui, B. Liu et al., “Atorvastatin protects vascular smooth muscle cells from TGF- $\beta 1$-stimulated calcification by inducing autophagy via suppression of the $\beta$-catenin pathway," Cellular Physiology and Biochemistry, vol. 33, no. 1, pp. 129-132, 2016.

[30] J. B. Koenig and I. Z. Jaffe, "Direct role for smooth muscle cell mineralocorticoid receptors in vascular remodelling: novel mechanisms and clinical implications," Current Hypertension Reports, vol. 16, no. 5, pp. 427-425, 2016.

[31] A. M. Thompson, K. A. Martin, and E. M. Rzucidlo, "Resveratrol induces vascular smooth muscle cell differentiation through stimulation of SirT1 and AMPK," PLoS One, vol. 9, no. 1, pp. 495-498, 2016.

[32] H. M. Liu, X. M. Li, F. Qin, and K. X. Huang, "Selenium suppresses oxidative-stress-enhanced vascular smooth muscle cell calcification by inhibiting the activation of the PI3K/AKT and ERK signalling pathways and endoplasmic reticulum stress," Journal of Biological Inorganic Chemistry, vol. 19, no. 3, pp. 375-388, 2016. 